\title{
ANÁLISE DE PROCESSOS POR MEIO DA SIMULAÇÃO \\ COMPUTACIONAL: PROPOSTA DE UMA ESTRATÉGIA DE ENSINO NA GRADUAÇÃO EM ADMINISTRAÇÃO
}

COMPUTER SIMULATED PROCESS ANALYSIS:

A PROPOSED TEACHING STRATEGY

FOR BUSINESS ADMINISTRATION DEGREES

\author{
Recebido em: 02/07/2012 Aprovado em: 10/08/2012 \\ Avaliado pelo sistema double blind review \\ NOELTORRES JÚNIOR noelface@gmail.com \\ Editora Científica: Manolita Correia Lima \\ GUILHERME GONÇALVES DE SOUZA \\ JÚLIAZANDOMENICO DO NASCIMENTO \\ UNIVERSIDADE FEDERAL DE MINAS GERAIS
}

\begin{abstract}
RESUMO
Dada a importância do uso da simulação computacional de eventos discretos para a análise e melhoria dos processos existentes nas organizações, este trabalho aborda o ensino desta técnica no curso de graduação em administração. O mesmo demonstra uma estratégia de ensino cuja ênfase recai na compreensão e análise do processo que será simulado em contraste com abordagens existentes, que privilegiam o conhecimento das rotinas e especificidades dos softwares de simulação. A abordagem proposta é baseada nos trabalhos de Laguna e Marklund (2004) e Hartvigsen (2005). A simulação computacional é feita por meio de planilhas eletrônicas, que utilizam o pacote SimQuick. Esta estratégia é composta de quatro etapas. A primeira consiste na identificação de oportunidades e definição do escopo do trabalho, a segunda visa uma descrição detalhada do processo que será analisado, a terceira consiste na avaliação do processo por meio da simulação computacional, e, finalmente, a última etapa consiste na proposta de redesenho. Um caso de modelagem realizado por um grupo de alunos é apresentado como recurso didático para esclarecer e demonstrar a estratégia de ensino proposta.

Palavras-chave: ensino de simulação em administração; análise de processos; simulação de eventos discretos.
\end{abstract}

\footnotetext{
ABSTRACT

Considering the importance of the use of discrete event simulation to analyze and improve existing processes within organizations, this paper discusses the teaching of this technique in undergraduate business administration courses. The paper outlines a teaching strategy where the emphasis lies on understanding and analyzing the process to be simulated in contrast to existing approaches that focus on knowing the routines and particularities of simulation software. The proposed approach is based on the works of Laguna and Marklund (2004) and Hartvigsen (2005). The simulation is carried out using spreadsheets and the SimQuick software package. This strategy consists of four steps. The first step comprises identifying opportunities and defining the scope of work, the second step aims at a detailed description of the process to be analyzed, the third step consists of the process evaluation through computer simulation, and finally, the last step encompasses the redesign proposal. A case modeling conducted by a group of students is presented here as a teaching resource to clarify and demonstrate the proposed teaching strategy.

Keywords: teaching simulation in management; process analysis; discrete event simulation.
} 


\section{INTRODUÇÃO}

O desenvolvimento da informática conduziu uma utilização generalizada de métodos de simulação e ferramentas em diversas áreas. Como exemplos deste fenômeno, pesquisadores, médicos e cirurgiões cada vez mais utilizam técnicas virtuais de operação antes de testar ou usá-las em pacientes reais; os simuladores são usados no treinamento de pilotos e no teste de manobras complicadas por navios ou aviões. A despeito disto, a melhoria na capacidade de processamento nos computadores não tem levado a desenvolvimentos semelhantes na área das ciências sociais. Nesta área, a simulação ainda é raramente utilizada por pesquisadores de gestão. Seu uso ainda fica restrito a problemas de otimização em sistemas de manufatura e logística (BERENDs; ROMME, 1999). No Brasil, os autores arriscam afirmar que o quadro não é muito diferente desta situação.

Abordagens voltadas para gestão por processos se fazem cada vez mais presentes no cotidiano das organizações. Estas abordagens por sua vez, suscitam o uso de métodos e ferramentas voltados à análise dos processos por parte de seus gestores (GONÇALVES, 2000; BARBARÁ, 2008). Como a modelagem computacional é um instrumento importante para o gerenciamento e a melhoria dos processos de negócio, o ensino destas técnicas pode ser visto como importante para cursos de administração. Entretanto, o ensino de simulação computacional ainda fica circunscrito a cursos de engenharias e áreas correlatas.

Alguns fatores podem ser entendidos como obstáculos à maior disseminação destas técnicas no ensino de graduação em administração. É importante citar alguns deles. Um fator relevante refere-se à complexidade de assimilação dos softwares de simulação de eventos discretos, objeto deste trabalho. Estes, geralmente, demandam substancial conhecimento de seus comandos e bibliotecas, fazendo com que o instrutor e seus alunos dediquem um tempo considerável da disciplina ou do curso no entendimento do programa, em detrimento do maior conhecimento acerca do processo que deverá ser simulado (BORN; STÅHL, 2008). Além destes aspectos, deve-se pesar 
o fato de o software ter seus comandos e menus em uma língua estrangeira. Outro obstáculo consiste no elevado preço de aquisição desses programas.

A despeito destas restrições, algumas iniciativas se contrapõem a estes obstáculos, possibilitando melhor divulgação e ensino destas técnicas, uma vez que novas abordagens para ensino e uso da simulação de eventos discretos são propostas. Nestas, a simulação computacional por ser realizada por meio de planilhas eletrônicas ou utilizando softwares mais amigáveis, simples e baratos (HILL, 2002). O enfoque maior fica na compreensão do processo, que será simulado, e no estudo das possibilidades de redesenho. A compreensão do software não ocupa mais o espaço central no ensino destas técnicas. Ståhl (2007) reporta a necessidade de se trabalhar com este enfoque, ao ensinar a simulação computacional de eventos discretos para estudantes de negócios e executivos.

Diante destas considerações, o problema de pesquisa concentrase na proposição de uma estratégia de ensino da técnica de simulação computacional de eventos discretos, que se concentre na compreensão do processo, que será simulado, e no estudo das possibilidades de redesenho, haja vista a importância desses aspectos para a análise e melhoria dos processos produtivos e a relevância deste tema na formação dos alunos dos cursos de graduação em administração.

Utilizando-se desta nova abordagem, na qual a ênfase da modelagem por meio de simulação computacional passa a ser a compreensão do processo, o presente trabalho demonstra a estratégia de ensino utilizada em uma disciplina do curso de graduação em Administração, conduzida em três diferentes turmas, ao longo dos últimos três anos. A abordagem proposta baseia-se, principalmente, nos trabalhos de Laguna e Marklund (2004) e Hartvigsen (2005). Nesta abordagem, o uso de métodos e ferramentas para a análise e compreensão do processo a ser modelado é privilegiado. Nesta direção, a simulação é utilizada como uma ferramenta, que apoia estas atividades e é feita por meio de planilhas eletrônicas utilizando-se o pacote SimQuick (Hartvigsen, 2005). Para tanto, este trabalho descreve a importância da análise e da simulação de processos; posteriormente, 
apresenta a estratégia de ensino construída dentro desta nova abordagem, e, finalmente, exibe um caso de modelagem realizado por um grupo de alunos, durante o ensino deste tema no curso de graduação em Administração. O caso apresentado é utilizado como recurso didático para esclarecer a estratégia de ensino proposta, uma vez que o mesmo demonstra todas as fases sugeridas por esta estratégia, realçando a importância da compreensão detalhada do processo para a proposição de alternativas de redesenho que estejam apoiadas na utilização de técnicas de simulação computacional. 


\section{ANÁLISE DE PROCESSOS E A SIMULAÇÃO}

As organizações podem ser entendidas como uma coleção de processos; nesta visão, todo trabalho realizado nas mesmas faz parte de algum processo (GONÇALVES, 2000). Johansson (I995) realça que a compreensão dos processos é a chave para o sucesso em qualquer negócio. Sendo os processos responsáveis pelo que é ofertado ao cliente, pode-se dizer que uma organização é tão efetiva quanto seus processos.

Neste contexto, as organizações devem conhecer e melhorar seus processos, continuamente, como uma estratégia para melhoria da qualidade e da produtividade do próprio negócio. Várias vantagens são obtidas com um bom gerenciamento dos processos: I) melhoria na utilização dos recursos; II) melhoria na comunicação entre as áreas; III) maior envolvimento dos funcionários; Iv) melhor avaliação e controle dos processos considerados críticos; v) foco no atendimento às necessidades dos clientes; vi) processos claramente definidos, com base em padrões de qualidade estabelecidos; viI) facilidade na implantação de mudanças; e viII) balanceamento entre as tarefas (REIS; BLATTMANN, 2004).

Para que estes benefícios sejam conseguidos, as organizações deverão adotar uma gestão orientada por processos. Para tanto, muitas deverão despender um grande esforço de mudança no sentido de adotar várias práticas voltadas a este fim (GONÇALVES, 2002). Uma atividade essencial para que organização caminhe nesta direção é o conhecimento dos processos existentes e a proposição de melhorias ou redesenho nos mesmos. Estas atividades dependem, em grande parte, do uso de métodos e técnicas de modelagem e da própria simulação computacional.

A modelagem consiste na representação simplificada de um dado sistema por meio de um modelo. Portanto, um modelo é uma abstração da realidade. Estes podem ser classificados como simbólicos ou matemáticos (BERENDs; ROMME, I999).

A modelagem dos processos pode ser feita por meio de modelos simbólicos, que utilizam diversas técnicas de representação gráfica. Algumas delas 
serão apresentadas no texto, e as mesmas serão utilizadas na estratégia de ensino, exposta neste trabalho.

Um método muito utilizado para a representação das etapas ou atividades do processo do serviço é o fluxograma. Nesta ferramenta, os atrasos, esperas, atividades de inspeção, operação e transporte são identificados, além de auxiliar na identificação de pontos problemáticos e pontos de intervenção para solução de problemas relacionados à qualidade (FItZsımmons; FITZSIMMONS, 20I0).

Processos que envolvem a participação do cliente podem ser representados pelo blueprinting do serviço. Este consiste em um mapa que contém todas as transações entre o pessoal da linha de frente e os clientes externos. Este também mostra as evidências físicas observadas pelos clientes, quais atividades são realizadas mediante interação entre clientes e funcionários da linha de frente, e quais atividades são realizadas sem interação com os clientes externos. O blueprinting também auxilia na solução de problemas, na identificação dos pontos de possíveis fracassos e pode destacar oportunidades de melhoria (BITNER; OSTROM; MORGAN, 2008).

Os processos podem também ser modelados por meio do diagrama PERT, que consiste em um diagrama de rede formado de nós e arcos, que representam as relações entre as atividades. Esse diagrama é útil na estimação do tempo de processamento e na visualização de relações de precedência nas atividades existentes no processo (LAGUNA; MARKLUND, 2004).

A Simulação pode ser definida como a construção e manipulação operacional de um modelo, isto é, uma representação física ou simbólica de todos ou de alguns aspectos de um processo físico e ou social (BERENDs; ROMME, 1999).

Os sistemas reais apresentam maior complexidade que os modelos, pois estes primeiros mudam seu estado, ao longo do tempo, e agem segundo uma natureza aleatória. Diante da existência destas características, a simulação computacional torna-se uma alternativa adequada de modelagem, na medida em que consegue capturar com mais fidelidade o comportamento do sistema real (MEDINA; CHWIF, 2007). 
A simulação computacional pode ser classificada em três categorias básicas: simulação de Monte Carlo, Contínua e de Eventos Discretos. A simulação de Monte Carlo utiliza geradores de número aleatórios para simular, não considerando explicitamente o tempo como uma variável. Entretanto, a simulação de eventos discretos e a simulação de eventos contínuos levam em consideração a mudança de estado do sistema ao longo do tempo (MEDINA; CHWIF, 2007).

De maneira geral, a simulação computacional apresenta diversas vantagens para a análise dos processos pelos seus gestores. São exemplos: - Em muitos casos, o desenvolvimento de um modelo analítico matemático para analisar o processo é extremamente difícil de ser operacionalizado, tornando a simulação a melhor alternativa de modelagem para este processo; - Em muitos casos, o processo apresenta um comportamento dinâmico no tempo, modificando seu estado durante sua operação, tornando necessária incorporação da variabilidade no modelo que irá analisar o mesmo. Nesta situação, a simulação possibilita a incorporação satisfatória desta variabilidade no modelo;

- Simulações fornecem um ambiente seguro para se cometer erros e, consequentemente, para aprender com os mesmos;

- Simulações possibilitam a experimentação e a verificação de diferentes cenários para o processo em análise;

- Pelas suas características de maior semelhança com o modelo real, a simulação permite maior aprendizagem por parte dos gestores (MAHBOUBIANA, 20Io; LAGUNA; MARKLUND, 2004).

Existe uma grande quantidade de softwares que podem ser utilizados na simulação de processos. Uma lista de software livres de simulação pode ser encontrada no endereço eletrônico http://www.topology.org/soft/sim. html (HILL, 2002).

Apesar de ser fortemente ignorada por especialistas em modelagem, a planilha eletrônica fornece um ambiente diversificado e robusto para a maioria das necessidades de modelagem. Essa planilha provê meios para armazenar e gerenciar dados, executar análises estatísticas, conduzir 
importação e exportação de tabelas e gráficos, além de executar a interface com outros softwares mais poderosos (HILL, 2002).

O uso da planilha eletrônica no ensino de métodos quantitativos de gestão tem cada vez se tornado mais presente. A familiarização preexistente com esta plataforma proporciona vários benefícios para os alunos e o instrutor no ensino de uma dada técnica. Entretanto, diante de diferenças entre o software tradicional de simulação e a planilha eletrônica, os usuários devem realizar algumas adaptações no processo de modelagem (HILL, 2002).

Levando-se em consideração o custo de aquisição e a facilidade de aprendizagem, optou-se pela utilização do pacote SimQuick (http://www. prenhall.com/hartvigsen), que é uma aplicação do software Excel e permite a realização de simulação de processos dentro do ambiente de planilha Excel. SimQuick não é um suplemento do Excel (como um .xla); é um modelo de planilha no qual um usuário especifica os elementos de um modelo de simulação, a parametrização desses elementos, e as conexões entre os mesmos. Seu funcionamento pode ser compreendido em poucas horas de aulas. Pode ser descrito como um pacote de primeira geração. Portanto, apresenta varias limitações se comparado com softwares maduros e dedicados como o Extend, ARENA, ProModel. No entanto, mesmo diante das suas limitações, o SimQuick é bastante adequado como ferramenta para entender o funcionamento e as características dos processos. Pode ser visto como uma ferramenta inicial de simulação para análise de processos. Possibilita a estimação da capacidade, o tempo de espera, o tamanho da fila e o grau de utilização dos recursos, além de possibilitar a realização de análises estatísticas destas variáveis. Este software é utilizado em vários cursos de mBa nos Estados Unidos (LEV, 2002; HILL, 2002; HARTVIGSEN, 2005).

No SimQuick, a configuração do modelo de simulação é feita mediante o uso de alguns blocos de construção. O programa trabalha com cinco tipos de blocos. São eles:

- Bloco "Buffers": Funciona como uma entidade de estocagem no processo; - Bloco "Entrances": Entidade existente para definir o processo de chegada de produtos ou clientes; 
- Bloco "Work Stations": Esta entidade funciona como uma estação de trabalho que processa os produtos ou as pessoas em um processo;

- Bloco "Decision Points": Esta entidade é utilizada quando o objeto a ser processado pode seguir em mais de uma direção no processo;

- Bloco "Exits": Esta entidade é utilizada quando o objeto já processado deixa o processo, segundo um escala de tempo ou segundo uma distribuição estatística (HARTVIGSEN, 2005).

O preenchimento dos blocos de construção existentes no SimQuick se dá mediante o acionamento dos "botões de links" existentes no Painel de Controle, como mostra o exemplo deste painel na Figura r. Cada "botão de link" conduz a uma planilha específica, que deverá ser preenchida com os parâmetros pertinentes ao modelo e ao bloco de construção (HARTVIGSEN, 2005).

Figura 1 Painel de controle do SimQuick

\section{SimQuick}

\section{Control Panel}

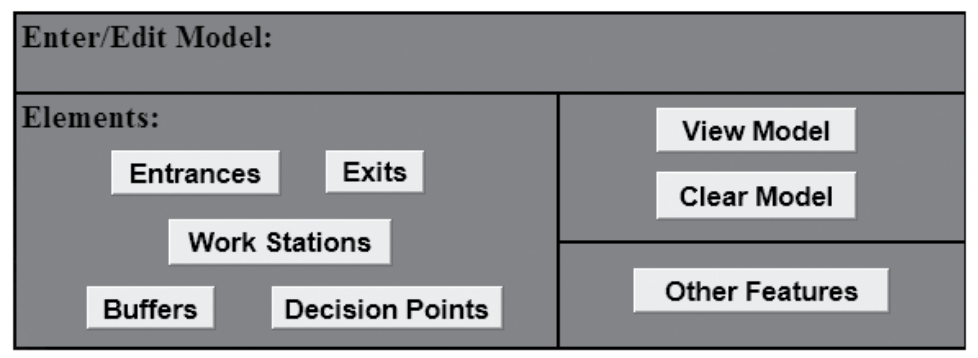

\begin{tabular}{|l|c|l|}
\hline \multicolumn{2}{|l|}{ Simulation controls: } & View Results \\
\hline Time units per simulation $₫$ & 18000 & \multirow{2}{*}{ Simulation(s) completed! } \\
\cline { 1 - 2 } Number of simulations $₫$ & 50 & \\
\hline Run Simulation(s) & & \\
\hline
\end{tabular}




\section{PROCEDIMENTOS METODOLÓGICOS}

Este texto visa demonstrar uma estratégia de ensino desenvolvida pelos autores e apoiada nos trabalhos de Laguna e Marklund (2004) e Hartvigsen (2005) para a análise de processos, apoiada na técnica de simulação computacional de eventos discretos. Esta estratégia foi aprimorada pela experiência docente de um dos autores, que a utilizou em três turmas de uma disciplina optativa dedicada a este fim, no curso de graduação em administração de uma dada universidade pública, durante os últimos três anos. Por ter como principal finalidade a demonstração de uma dada estratégia de ensino, este trabalho é do tipo exploratório e de natureza descritiva.

Nesta estratégia, a análise de processos foi feita segundo um conjunto de atividades. Estas atividades podem ser agrupadas em quatro etapas onde, de maneira gradual, conceitos e ferramentas acerca da análise de processo são apreendidos e utilizados pelos alunos. Para tanto, durante o curso e como produto final da disciplina, os alunos deveriam realizar um trabalho prático, que percorre todas as quatro etapas propostas. São elas:

- Primeira etapa - Identificação de oportunidades e definição do escopo do trabalho: por meio de entrevistas aos seus gestores, o grupo de alunos deverá escolher uma organização, identificando oportunidades de melhoria na mesma. Para tanto, eles deverão reconhecer quais os principais processos existentes na organização investigada e selecionar alguns. Deverão ser mapeados para cada um desses processos os principais recursos, produtos e clientes. Como cada um desses processos, normalmente, está envolvido na geração de valor para os clientes externos ou internos, eles deverão realizar uma série de perguntas visando à identificação de oportunidades de melhoria sob esta perspectiva. Além disso, a escolha do processo que será analisado deverá considerar a facilidade de acesso aos dados e às informações. Uma vez selecionado o processo, os limites dessa análise devem ser claramente estabelecidos.

- Segunda etapa - Documentação do Processo: uma vez que o escopo do processo a ser analisado foi estabelecido, o grupo deverá realizar o 
mapeamento detalhado do mesmo. Nesta etapa, uma ou mais ferramentas de diagramação podem ser utilizadas. Dados referentes ao tempo de processamento de cada atividade devem ser levantados e estimados. De posse destas informações, realiza-se uma avaliação do tempo total de processamento, do fluxo de produção e da capacidade de processamento. Estas informações serão posteriormente confrontadas com as estatísticas obtidas pela simulação computacional.

- Terceira etapa - Avaliação do Processo por meio da Simulação Computacional: uma vez que o processo foi diagramado e o tempo de cada atividade foi estimado, pode-se proceder com a construção do modelo no software de simulação. Com o modelo do processo atual já configurado e verificado no software de simulação, pode-se proceder com a análise do processo propriamente dito. Esta pode ser feita tanto pela modificação da configuração do processo como pelo teste do impacto destas mudanças no tempo de processamento, no tempo de espera, no grau de utilização dos recursos e na capacidade de processamento.

- Quarta etapa - Proposta de Redesenho: esta etapa consiste numa análise cuidadosa do processo e de seu desempenho com os indicadores obtidos com o Software de Simulação, o que deverá ser feito utilizando um pensamento analítico e criativo. Uma lista de ideias para aperfeiçoamentos deve ser elaborada; em seguida, estas ideias devem ser filtradas e reavaliadas. A proposta final dever ser documentada e conter uma descrição das mudanças propostas e do ganho obtido.

O Quadro I apresenta a síntese das etapas e dos principais conceitos e ferramentas que devem ser utilizados em cada uma delas. 
Quadro 1 Síntese das etapas e dos principais conceitos e ferramentas utilizados

\begin{tabular}{|c|c|}
\hline Etapa & Principais ferramentas e conceitos utilizados e a referência utilizada \\
\hline $\begin{array}{l}\text { Identificação de } \\
\text { oportunidades e } \\
\text { definição do escopo } \\
\text { do trabalho }\end{array}$ & $\begin{array}{l}\text { Processos - conceito e tipos (GONÇALVES, 2000). } \\
\text { Cadeia de Valor (PAIM et al., 2009; BARBARÁ, 2008). } \\
\text { Fluxograma (FITZSIMMONS; FITZSIMMONS, 2010). } \\
\text { Blueprinting do serviço (BITNER; OSTROM; MORGAN, 2008). }\end{array}$ \\
\hline $\begin{array}{l}\text { Documentação do } \\
\text { Processo }\end{array}$ & $\begin{array}{l}\text { Diagrama de rede; Lei de Little e suas aplicações; } \\
\text { Mensuração do tempo e da capacidade produtiva (LAGUNA; MARKLUND, } \\
\text { 2004). }\end{array}$ \\
\hline $\begin{array}{l}\text { Avaliação do } \\
\text { Processo por meio } \\
\text { da Simulação } \\
\text { Computacional }\end{array}$ & $\begin{array}{l}\text { Estatística descritiva; distribuições de probabilidade; testes de aderência; } \\
\text { verificação de modelos de simulação (MEDINA; CHWIF, 2007). } \\
\text { Software SimQuick (HARTVIGSEN, 2005). }\end{array}$ \\
\hline $\begin{array}{l}\text { Proposta de } \\
\text { Redesenho }\end{array}$ & $\begin{array}{l}\text { Perdas em Processos (LAGUNA; MARKLUND, 2004). } \\
\text { Práticas de Redesenho (HARMON, 2007; REIJERSA; MANSARB, 2005). }\end{array}$ \\
\hline
\end{tabular}




\section{DEMONSTRAÇÃO DO CASO DE ANÁLISE DE PROCESSOS}

Com intuito de demonstrar a estratégia metodológica utilizada para análise de processos por meio da simulação computacional, esta seção descreve à aplicação das quatro etapas propostas em uma empresa logística. Esta intervenção foi feita por um grupo de alunos durante uma disciplina de análise de processos do curso de graduação em Administração. A empresa analisada é um operador logístico que atua no transporte rodoviário de bens para a região sudeste do Brasil.

\section{PRIMEIRA ETAPA-IDENTIFICAÇÃO DE OPORTUNIDADES E DEFINIÇÃO DO ESCOPO DO TRABALHO}

Nesta etapa, um grupo de alunos visitou a empresa para conhecer o ambiente de trabalho e os processos existentes na mesma. Como um dos membros do grupo atuava como estagiário nesta organização, foi possível obter várias informações detalhadas sobre os processos existentes. Duas entrevistas foram realizadas. A primeira se deu com o diretor da área de planejamento. Esta foi decisiva para o andamento deste trabalho, uma vez que este diretor possibilitou o acesso a vários dados utilizados neste trabalho.

Após esta entrevista e considerando sua importância para a organização estudada, o grupo escolheu o setor de expedição de documentos para a realização do estudo, uma vez que o mesmo é responsável pela documentação legal dos serviços prestados.

Após essa definição, o segundo entrevistado foi um funcionário do setor de expedição que ajudou o grupo a ter um melhor conhecimento das atividades realizadas nesta área.

Foi feito um Macro Fluxograma e um Diagrama Blueprinting desta empresa, e estes são apresentados nas Figuras 2 e 3 deste trabalho. 
Figura 2 Macro Fluxograma da empresa

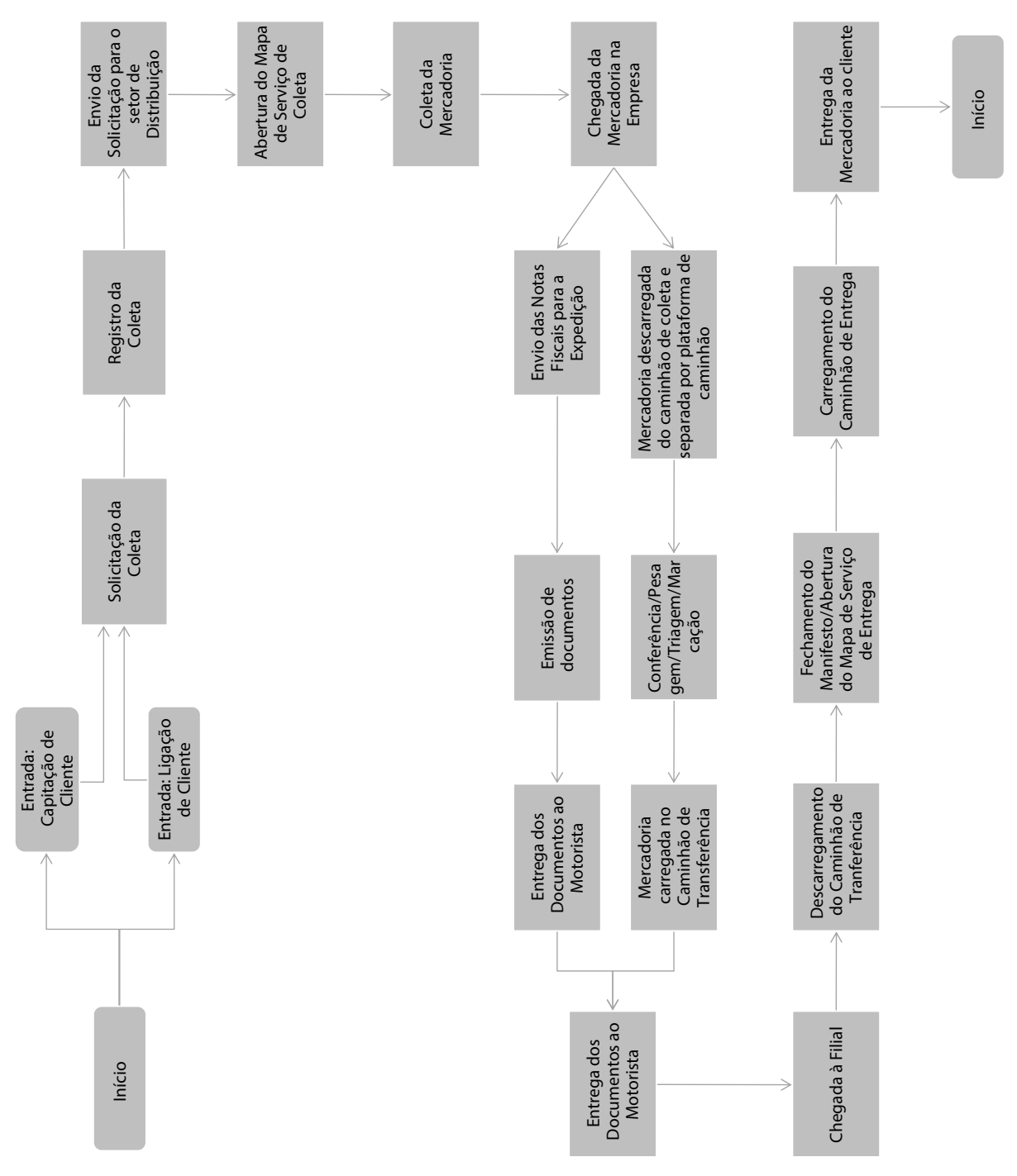


ANÁLISE DE PROCESSOS POR MEIO DA SIMULAÇÃO COMPUTACIONAL: PROPOSTA DE UMA ESTRATÉGIA DE ENSINO NA GRADUAÇÃO EM ADMINISTRAÇÃO

Figura 3 Blueprinting da empresa analisada

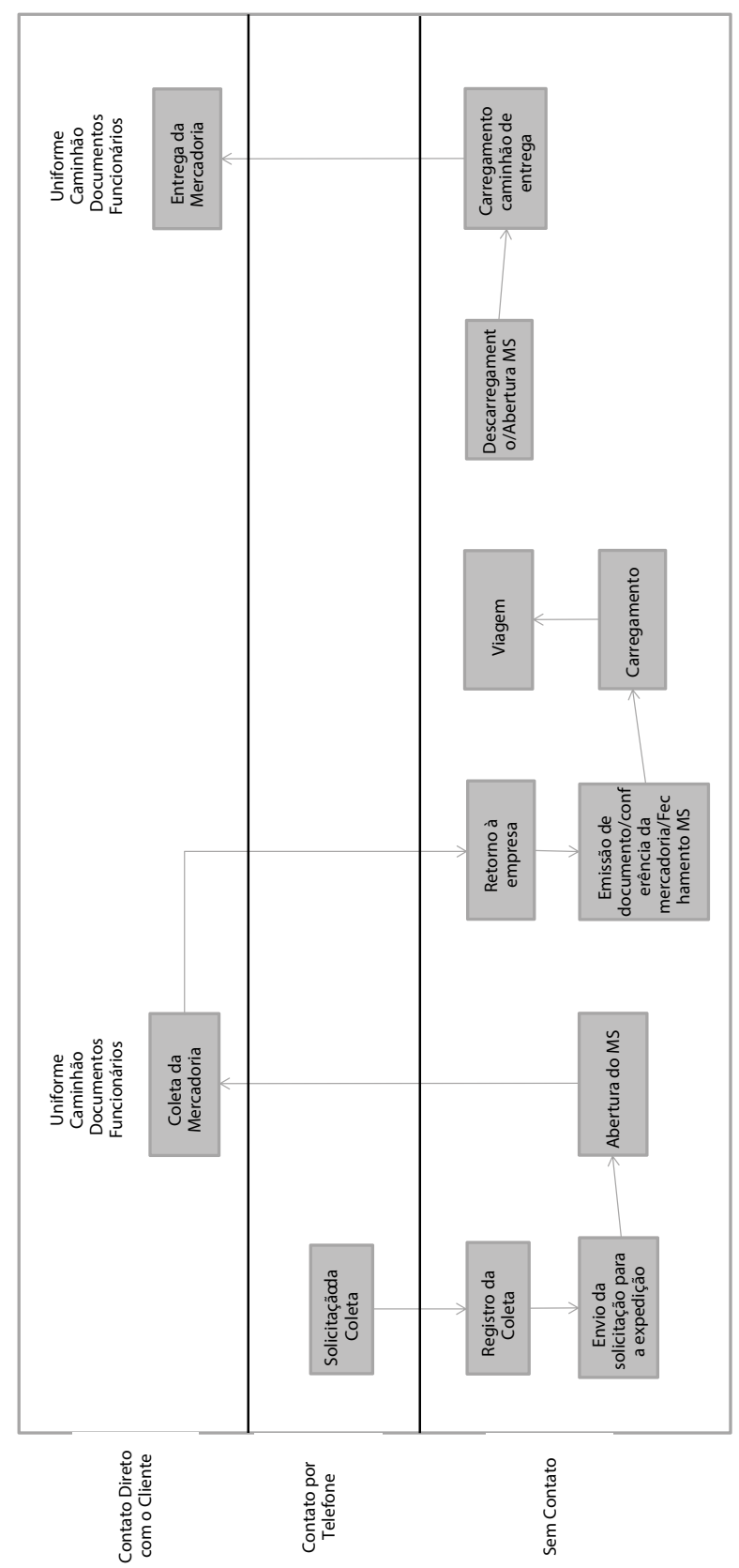


Basicamente a função do setor de expedição é emitir e organizar os documentos fiscais necessários para a prestação de serviços de transporte. Isso inclui: emissão do Conhecimento de Transporte Rodoviário de Carga (CTRC), emissão do Recibo de Pagamento para Autônomo (RPA), emissão do Manifesto de Carga (Manifesto), emissão da Solicitação de Monitoramento Preventivo (SMP) e organização dos documentos impressos na ordem exigida pela lei (incluindo as notas fiscais dos produtos).

A análise da entrevista realizada com o gestor desta organização apontou a necessidade de redução da hora extra neste setor. Nesta direção, realizouse um levantamento histórico das entradas e saídas dos funcionários. Para tanto, utilizaram-se os dados registrados pelo cartão de ponto no período de 16 de fevereiro de 2012 a I5 de abril de 20I2. Estes dados revelaram que:

- Em vários dias, alguns funcionários chegaram à empresa, em horários antecipados, gerando um custo adicional por hora extra. Existiram também casos de horas extras após o horário normal de expediente;

- Por se tratar de um trabalho noturno, em que os documentos devem ser elaborados o mais rapidamente possível para a expedição das mercadorias, que chegaram durante o dia, em alguns dias, os funcionários saíram mais cedo do expediente, uma vez que todas as tarefas daquele dia foram concluídas antes do término do horário normal. Como não há um desconto no salário do funcionário, este evento acaba gerando um custo desnecessário para a organização.

- Há um gasto com pagamento de adicional noturno, que ocorre devido ao fato de os funcionários trabalharem após as $22 \mathrm{~h}$.

Diante destes fatos, o grupo entendeu que o problema a ser estudado no processo consiste na redução de custos com os funcionários existentes nos processos de expedição realizados pela equipe do turno noturno.

\section{SEGUNDA ETAPA - DOCUMENTAÇÃO DO PROCESSO}

Uma vez que o escopo do processo foi definido, realizou-se um mapeamento detalhado do mesmo.

O setor de expedição conta com seis funcionários entre os quais um trabalha no turno diurno ( 9 h às $15 \mathrm{~h}$ ) e os outros cinco no turno noturno. 
Dos que trabalham à noite, tem-se um coordenador e quatro auxiliares de expedição. O coordenador e um dos auxiliares chegam às i hh e os outros três auxiliares chegam às $\mathrm{I} 8 \mathrm{~h}$. Todos trabalham de segunda a sexta-feira, seis horas por dia, e realizam revezamento para alguns deles trabalharem aos sábados e nos domingos.

Ocorre uma divisão de tarefas dentro do setor. O coordenador é responsável principalmente pela emissão do SPM, RPA e Manifesto. Dois auxiliares fazem a emissão do CTRC e Manifestos de Cargas e os outros dois são responsáveis pela conferência e organização dos documentos para viagem. Por escolha dos integrantes do setor, há uma rotatividade nas tarefas realizadas pelos auxiliares.

Foi feito um fluxograma do processo de expedição que é mostrado na Figura 4 deste trabalho. 
Figura 4 Fluxograma do processo de expedição de notas fiscais

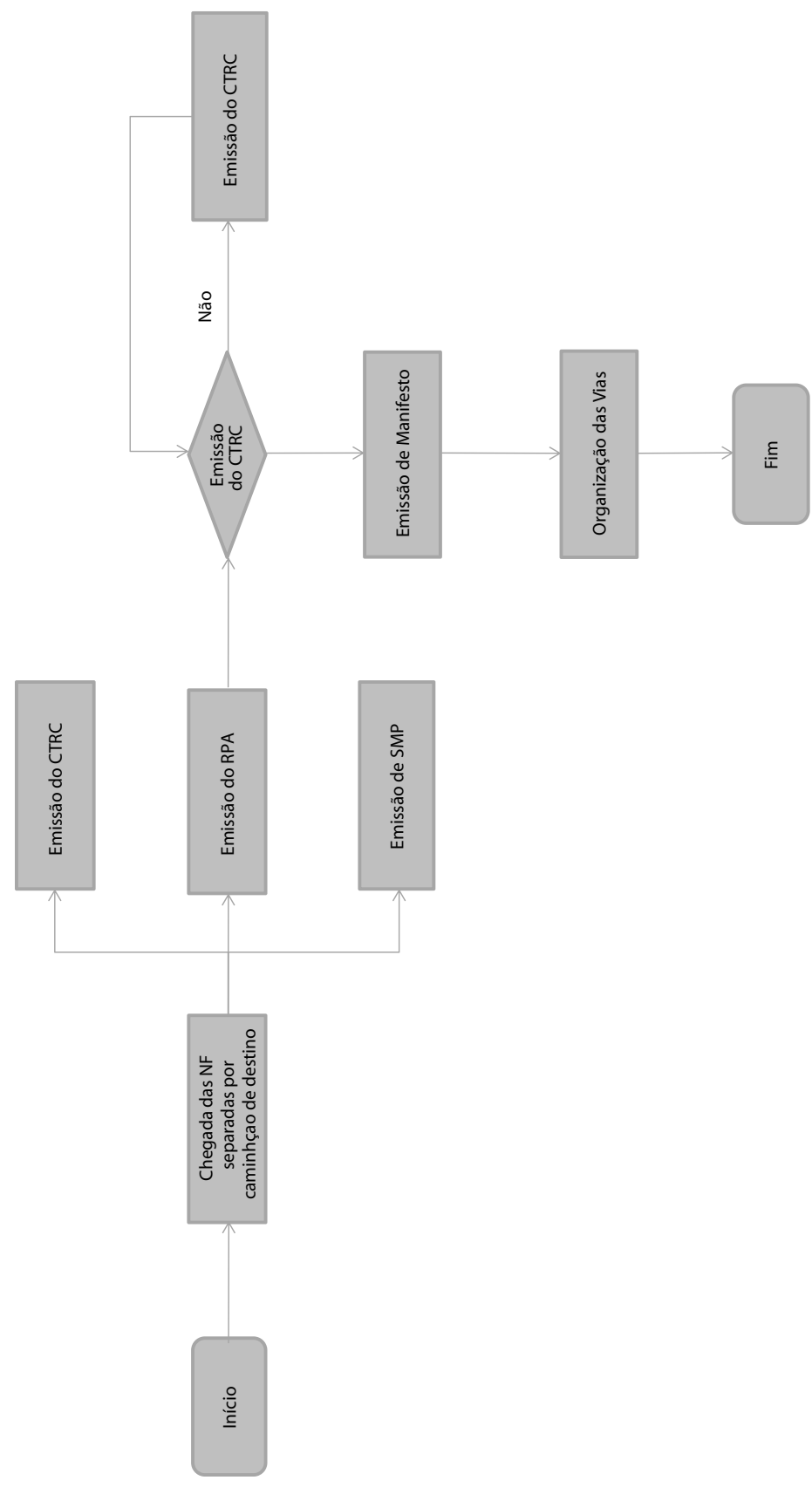


Com o objetivo de compreender melhor o processo apresentado na Figura 4, uma análise do tempo gasto por cada tipo de atividade foi feita. Para tanto, um diagrama de PERT foi elaborado e o mesmo é mostrado na Figura 5. Este diagrama baseou-se em tempos médios.

Figura 5 Diagrama de PERT do processo de expedição de documentos

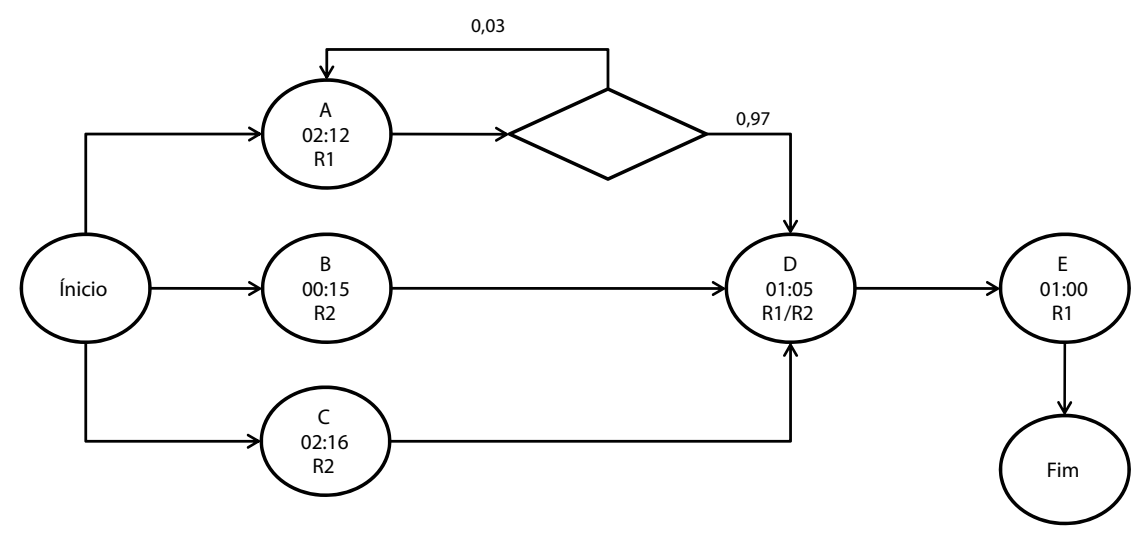

\begin{tabular}{|c|c|c|c|c|}
\hline \multicolumn{5}{|c|}{ Descrição das Atividades } \\
\hline Código & Descrição & Recursos & $\begin{array}{l}\text { Tempo de } \\
\text { Execução (min) }\end{array}$ & $\begin{array}{l}\text { Antecessoras } \\
\text { Imediatas }\end{array}$ \\
\hline A & Emissão de CTRC & $\mathrm{R} 1$ & $2: 12$ & - \\
\hline B & Emissão de RPA & $\mathrm{R} 2$ & $0: 15$ & - \\
\hline C & Emissão de SMP & $\mathrm{R} 2$ & $2: 06$ & - \\
\hline $\mathrm{D}$ & Emissão de Manifesto & $\mathrm{R} 1 / \mathrm{R} 2$ & $1: 05$ & $A, B, C$ \\
\hline$E$ & Organização das Vias & $\mathrm{R} 1$ & 1:00 & $\mathrm{D}$ \\
\hline
\end{tabular}

\section{Descrição dos Recursos}

\begin{tabular}{|c|c|c|}
\hline Código & Descrição & Quantidade de Recurso \\
\hline R1 & Auxiliar & 4 \\
\hline R2 & Coordenador & 1 \\
\hline
\end{tabular}


Baseando-se no diagrama de PERT apresentado na Figura 5 e na demanda média diária para cada tipo de documento gerado neste processo (CTRC, RPA, smp e Manifesto de Carga), calculou-se o tempo médio diário gasto por cada funcionário na realização das atividades. Estes Dados são apresentados na Tabela I.

Tabela 1 Cálculo do tempo teórico gasto diário por funcionário em cada documento

\begin{tabular}{|c|c|c|c|c|c|}
\hline Documento & $\begin{array}{l}\text { Recurso Princi } \\
\text { (Cp) }\end{array}$ & & $\begin{array}{l}\text { Carga de } \\
\text { Trabalho Tp } \\
\text { (minutos } \\
\text { por item) }\end{array}$ & $\begin{array}{l}\text { Demanda diária } \\
\text { (unidades) }\end{array}$ & $\begin{array}{l}\text { Tempo } \\
\text { Teórico } \\
\text { Gasto por } \\
\text { Funcionário } \\
\text { (minutos) }\end{array}$ \\
\hline CTRC & Auxiliar & 2 & $2,200 \times 1,03$ & 290 & 328,57 \\
\hline RPA & Coordenador & 1 & 0,250 & $\begin{array}{l}\text { mínimo de } 0 \\
\text { máximo de } 15\end{array}$ & $\begin{array}{l}0 \\
3,75\end{array}$ \\
\hline SMP & Coordenador & 1 & 2,100 & $\begin{array}{l}\text { mínimo de } 0 \\
\text { máximo de } 15\end{array}$ & $\begin{array}{l}0 \\
31,5\end{array}$ \\
\hline Manifesto de Carga & $\begin{array}{l}\text { Auxiliar/ } \\
\text { Coordenador }\end{array}$ & 5 & 1,083 & 15 & 3,2 \\
\hline Via Organizada & Auxiliar & 2 & 1,000 & 15 & 7,5 \\
\hline
\end{tabular}

Com relação aos dados coletados, faz-se necessária a apresentação de algumas observações e esclarecimentos:

- Para este estudo foram considerados os dados dos auxiliares que realizaram digitação do conhecimento analítico em grandes escalas, dessa forma o coordenador do setor não foi analisado. O quarto auxiliar não foi considerado para a média, pois em parte do período analisado estava de férias e, no tempo restante, não foi digitada uma quantidade significativa de CTRCs.

- No período analisado a empresa emitiu em média I5 Manifestos de carga por dia. Observou-se que um operador demora em média I minuto e 5 segundos para a operação de um manifesto (média feita com base em I2 emissões de um funcionário). De acordo com a Tabela I deste trabalho, o tempo teórico gasto por funcionário na digitação de Manifesto de Carga é 
de 3,2 minutos, o que, se comparado ao tempo total de trabalho, mostra-se irrelevante.

- O tempo de emissão de RPA e smp também é irrelevante já que, salvo exceções, estes documentos são emitidos pelo coordenador. Além disso, estão em menor quantidade que os Manifestos e levam um tempo médio de I5 segundos e 02 minutos e 06 segundos respectivamente (médias calculadas com base em 4 e II emissões respectivamente). Como pode ser visto na Tabela I deste trabalho, o tempo teórico gasto por funcionário para estas atividades é relativamente pequeno se comparado ao tempo de digitação de CTRCs.

- Sábados e domingos foram desconsiderados devido ao número reduzido de funcionários e tarefas a serem realizadas.

Diante das considerações acima, o estudo concentrou sua análise no processamento das CTRCs. Nesta direção, buscou-se conhecer melhor este processo. Para tanto, foi feito um estudo da utilização do tempo médio trabalhado. Uma síntese deste estudo é mostrada no Gráfico i deste trabalho e esta aponta a existência de oportunidades de melhoria de custo que serão exploradas na terceira etapa desta intervenção.

Gráfico 1 Síntese dos dados coletados referentes ao tempo trabalhado por cada funcionário

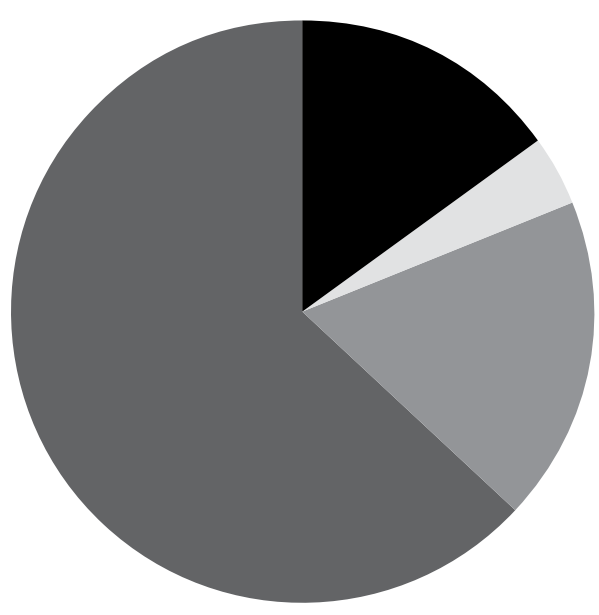

$63 \%$ Hora Normal

$18 \%$ Abono

4\% Hora Extra

$15 \%$ Ad. Noturno 


\section{TERCEIRA ETAPA - AVALIAÇÃO DO PROCESSO POR MEIO DA SIMULAÇÃO COMPUTACIONAL}

Uma vez que a análise será feita no processamento das CTRCs, buscou-se definir um modelo conceitual do processo que retratasse as atividades do processo atual, segundo a lógica utilizada pelo programa SimQuick. Este modelo é apresentado na Figura 6 a seguir.

Figura 6 Modelo conceitual do processo atual utilizando o SimQuick.

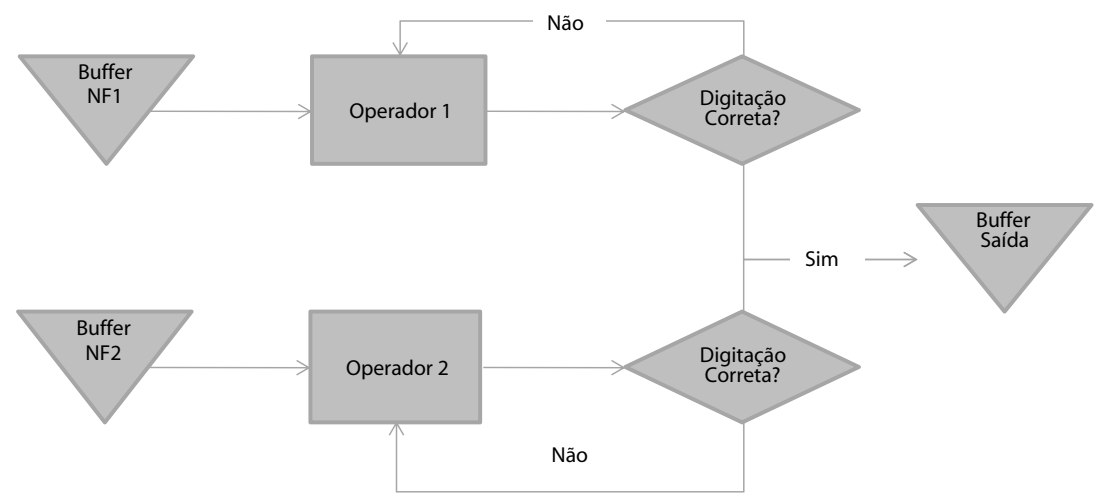

Uma vez definido o modelo procedeu-se à definição dos dados de entrada do mesmo. Para tanto, realizou-se uma análise estatística do tempo de processamento dos operadores. Esta análise revelou dois grupos distintos de operadores. No primeiro grupo, encontram-se os operadores experientes com um grau de destreza superior ao segundo grupo. Este segundo grupo é constituído por operadores com uma experiência menor nesta atividade, portanto realizam as atividades de emissão de CTRCs gastando um tempo maior. A taxa de processamento do primeiro grupo pode ser expressa por uma distribuição normal com média de I03 segundos e desvio padrão de 19 segundos por CTRCs. Já a taxa de processamento do segundo grupo pode ser expressa por uma distribuição normal com média de I4I segundos e desvio padrão de 3I segundos por CTRCs. A análise dos dados revelou também uma diferença no percentual retrabalhado. Funcionários do primeiro grupo retrabalham cerca de $2 \%$ dos documentos, enquanto os funcionários do 
segundo grupo retrabalham bem mais, cerca de $4 \%$ dos documentos. Como a média diária de CTRCs enviadas para o setor é da ordem de 290 e os funcionários não compartilham do mesmo pacote de notas, cada operador inicia a simulação com I45 unidades. Nesta configuração considerou-se que todas as notas fiscais já estão disponíveis no início do período de trabalho.

Após a definição do modelo conceitual do processo e dos parâmetros de entrada, procedeu-se com a simulação propriamente dita. Alguns resultados obtidos com a mesma são apresentados a seguir.

Gráfico 2 Boxplot das horas diárias trabalhadas por operador

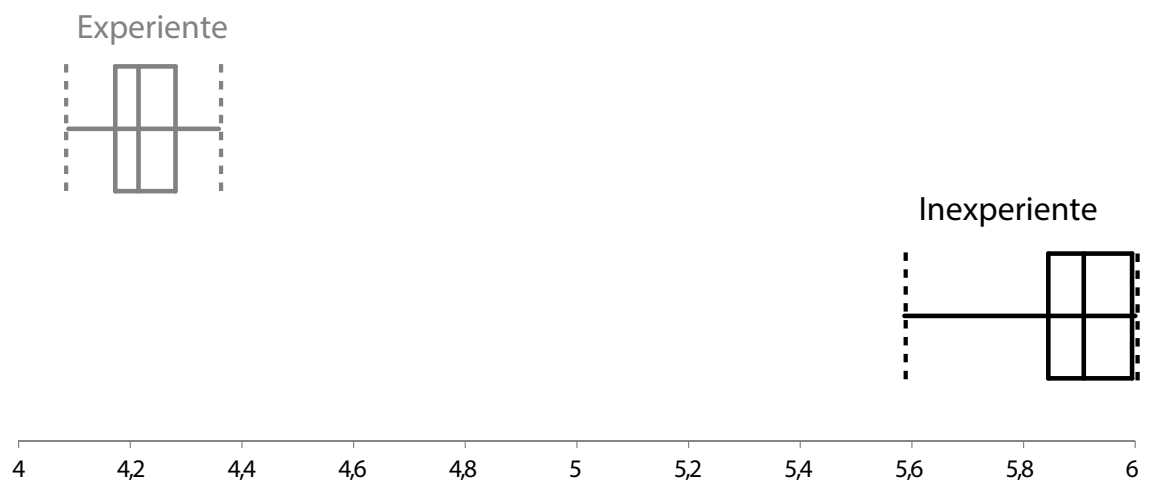

Gráfico 3 Boxplot do percentual retrabalhado por operador

Percentual de Retrabalho no Processo Atual

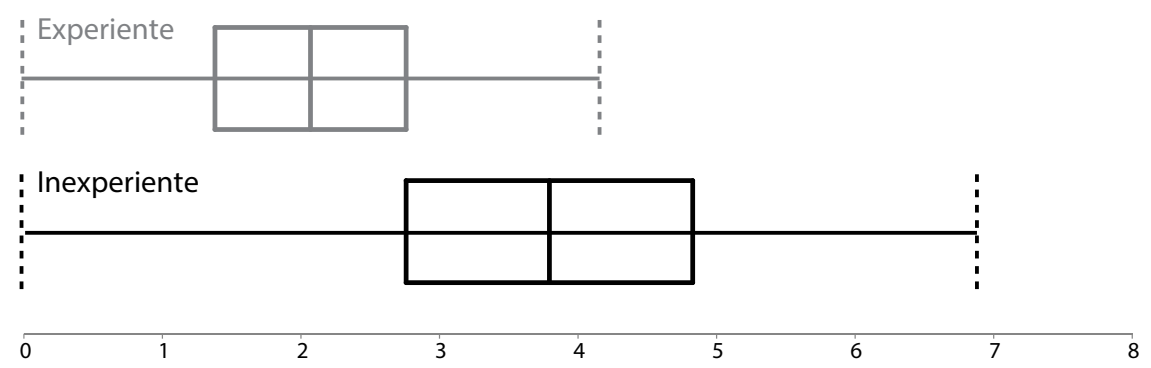


O Gráfico 3 revela a variação do percentual retrabalhado por cada operador. Apesar de o percentual médio de retrabalho ser maior para o funcionário pouco experiente, os dois funcionários apresentam variação semelhante.

Com base nestes dados, os custos com mão de obra deste processo foram estimados e são apresentados na Tabela 2.

Tabela 2 Custos com mão de obra do processo atual

\begin{tabular}{|c|c|c|}
\hline Descrição & \multicolumn{2}{|c|}{$\begin{array}{l}\text { Situação Atual: } 1 \text { funcionário pouco experiente e } 1 \text { funcionário } \\
\text { experiente trabalhando } 6 \text { horas por dia e iniciando o expediente } \\
\text { às } 18 \mathrm{~h} \text {. }\end{array}$} \\
\hline Tipo de gasto & $1^{\circ}$ Funcionário (Pouco Experiente) & 2 Funcionário (Experiente) \\
\hline Hora normal trabalhada & $\mathrm{R} \$ 580,00$ & $R \$ 420,00$ \\
\hline Hora extra trabalhada & $R \$ 0,00$ & $\mathrm{R} \$ 0,00$ \\
\hline Adicional noturno & $\mathrm{R} \$ 36,00$ & $\mathrm{R} \$ 4,00$ \\
\hline Abono & $R \$ 20,00$ & $R \$ 180,00$ \\
\hline Total por funcionário & $R \$ 636,00$ & $\mathrm{R} \$ \mathbf{6 0 4 , 0 0}$ \\
\hline Total gasto & $\mathrm{R} \$ 1.240,00$ & \\
\hline
\end{tabular}

Uma análise dos dados já levantados acerca do processo apontou algumas variáveis que poderiam impactar positivamente na redução dos custos. Vale aqui ressaltar algumas delas:

- O grau de experiência do operador influencia no tempo gasto no processamento dos documentos e na quantidade retrabalhada, consequentemente impacta no gasto com a mão de obra;

- A hora de início do turno de trabalho pode influenciar o gasto com adicional noturno e abono despendidos, portanto também impacta no gasto com a mão de obra;

- A duração do turno também influencia o valor gasto com os funcionários.

Levando-se em consideração estas variáveis, procedeu-se varias simulações testando diferentes cenários de configuração do processo. Para cada um deles calculou-se o custo médio com a mão de obra. Os resultados destas simulações estão apresentados na Tabela 3. 
Tabela 3 Custos com mão de obra com diferentes configurações

\begin{tabular}{|c|c|c|c|c|c|c|c|c|c|}
\hline 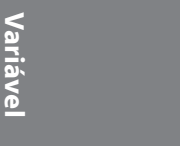 & $\frac{z}{c}$ & & 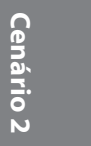 & & 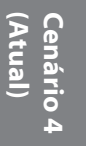 & 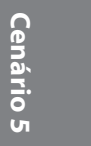 & 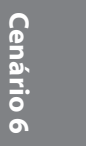 & है. & 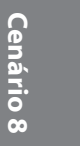 \\
\hline \multirow{2}{*}{$\begin{array}{l}\text { Inicio } \\
\text { do Turno }\end{array}$} & $17: 00 \mathrm{~h}$ & & $x$ & & & $\mathrm{x}$ & & $\mathrm{X}$ & \\
\hline & 18:00h & $x$ & & $\mathrm{x}$ & $x$ & & $x$ & & $\mathrm{x}$ \\
\hline \multirow{2}{*}{$\begin{array}{l}\text { Quantidade } \\
\text { de horas } \\
\text { trabalhadas }\end{array}$} & 6 & $x$ & $x$ & & $\mathrm{X}$ & $x$ & & $\mathrm{X}$ & \\
\hline & 5 & & & $x$ & & & $x$ & & $x$ \\
\hline \multirow{2}{*}{$\begin{array}{l}\text { Configuração } \\
\text { dos } \\
\text { funcionários }\end{array}$} & Experiente & & & & 1 & 1 & 1 & 2 & 2 \\
\hline & $\begin{array}{l}\text { Pouco } \\
\text { Experiente }\end{array}$ & 2 & 2 & 2 & 1 & 1 & 1 & & \\
\hline \multicolumn{2}{|c|}{ Custo total de mão-de-obra } & $\begin{array}{l}\text { RS } \\
1.272\end{array}$ & $\begin{array}{l}\mathrm{R} \$ \\
1.232\end{array}$ & $\begin{array}{l}\text { RS } \\
1.312\end{array}$ & $\begin{array}{l}\mathrm{R} \$ \\
1.240\end{array}$ & $\begin{array}{l}\text { RS } \\
1.216\end{array}$ & $\begin{array}{l}\text { R\$ } \\
1.160\end{array}$ & $\begin{array}{l}\text { RS } \\
1.200\end{array}$ & $\begin{array}{l}\text { RS } \\
1.010\end{array}$ \\
\hline
\end{tabular}

\section{QUARTA ETAPA - PROPOSTA DE REDESENHO}

Após a realização de diferentes cenários de simulação, procedeu-se à análise dos resultados e da verificação da aplicabilidade de cada um deles. Nesta direção, três cenários se mostraram promissores para a redução de custo.

Os cenários 6 e 8 apresentaram os menores custos. Entretanto alguns fatores impedem que eles sejam utilizados. Uma vez que os funcionários trabalhariam quase todo o tempo e, nessa situação, eles teriam cerca de Io०\% de ocupação, algo impossível de ser conseguido em uma situação real. Além disso, nestes cenários, os funcionários trabalhariam menos de 8 horas diárias. Nesta condição, os funcionários estão legalmente impossibilitados de receber horas extras, algo muito restritivo para o processo, pois impediria o mesmo de lidar com imprevistos.

O Cenário 7 se mostra a melhor alternativa. Uma vez que apresenta uma boa taxa de ocupação dos funcionários e possibilita uma margem de folga em termos de ocupação dos mesmos. Esta folga ajudaria a lidar com imprevistos 
no setor, uma vez que o tempo ocioso poderia ser eventualmente utilizado no processamento de CTRCs de pedidos que cheguem atrasados. Além disso, uma comparação do tempo gasto pelos funcionários (Gráfico 4) e o grau de retrabalho (Gráfico 5) deste cenário com a situação atual aponta uma melhoria significativa do processo nestes aspectos.

Gráfico 4 Boxplot das horas diárias trabalhadas nos processos

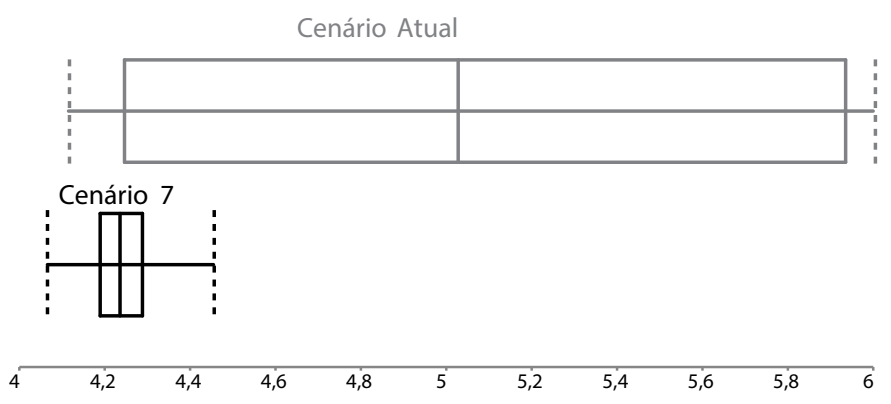

Gráfico 5 Boxplot do percentual retrabalhado nos dois processos

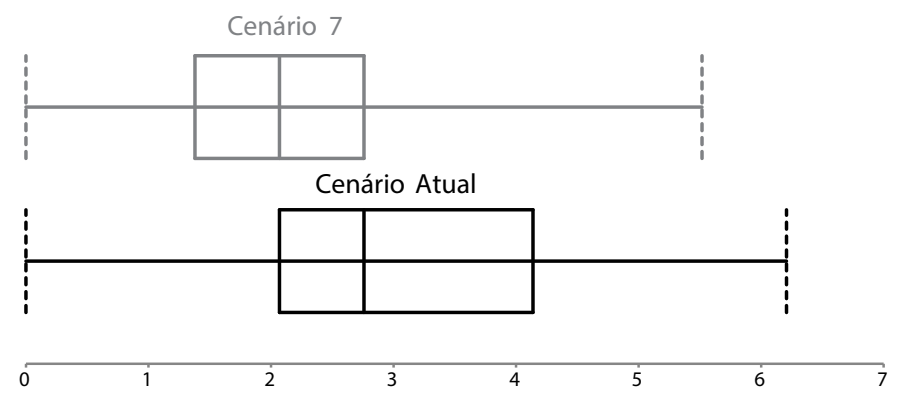




\section{CONSIDERAÇÕES FINAIS}

Considerando que o presente trabalho teve como objetivo a demonstração de uma estratégia de ensino da técnica de simulação computacional de eventos discretos voltados para a compreensão do processo e análise das possibilidades de seu redesenho pode-se concluir que este objetivo foi atendido. Esta constatação se baseia em alguns elementos. O primeiro deles refere-se ao fato de que, não obstante a estratégia proposta contemplar quatro etapas, apenas uma delas é dedicada à construção do modelo de simulação computacional. As demais etapas buscam a melhor compreensão do processo por meios de conceitos e ferramentas que possibilitam o entendimento dos processos envolvidos, dos objetivos e dos problemas enfrentados pela organização. Além disso, algumas destas ferramentas buscam melhor compreensão das etapas e das relações de precedência entre elas, possibilitando uma estimação inicial da capacidade e do tempo de processamento do processo focalizado pelo estudo. Como segundo elemento, pode-se afirmar que todo o esforço despendido para a análise do processo favorece a assimilação de práticas voltadas para a gestão por processos. Finalmente, devido à maior compreensão do processo, a escolha das variáveis que podem ser modificadas e a definição da melhor opção de redesenho ficam facilitadas com esta estratégia.

A despeito das vantagens apontadas anteriormente pelo estudo, por se tratar de uma pesquisa exploratória, essas não podem ser generalizadas para contextos diferentes do qual o trabalho foi conduzido. Neste sentido, os autores sugerem a realização de novos estudos em que se comparem as vantagens desta estratégia em relação à tradicional. Para tanto, duas turmas da mesma disciplina devem ser avaliadas. Em uma delas será utilizada a estratégia proposta pelo trabalho e na outra será utilizada a estratégia tradicional de ensino da técnica de simulação computacional.

A abordagem proposta se contrapõe à abordagem tradicional de ensino da simulação, no qual a ênfase está no domínio do software em detrimento do conhecimento e análise do próprio processo. Os trabalhos finais apresentados pelos alunos na disciplina que utilizaram esta abordagem 
proposta são animadores. A simulação de eventos discretos foi conduzida em diversos tipos de processos.

É importante frisar que o uso conjunto da simulação computacional e das técnicas de diagramação enriquece as atividades de redesenho de processos. Existe certa sinergia nestas duas ferramentas, uma vez que a diagramação dos processos possibilita entender as características do processo, bem como a modelagem computacional permite a criação de novos cenários, facilitando ao analista visualizar os impactos das alterações propostas.

A área de administração tem divulgado extensamente a necessidade das organizações repensarem seus processos e trabalharem com práticas voltadas para o conceito de gestão por processos. Nesta direção, faz-se necessária a divulgação de ferramentas que possibilitem aos administradores trabalharem neste propósito. O ensino e a divulgação da modelagem computacional de eventos discretos podem ser entendidos como elementos importantes para o atendimento deste fim. 


\section{REFERÊNCIAS}

BARBARÁ, S. (Org.). Gestão por processos: fundamentos, técnicas e modelos de implementação. 2.ed. Rio de Janeiro: Qualitymark, 2008,

BERENDS, P.; ROMME, G. Simulation as a Research Tool in Management Studies, European Management Journal, v. 17, n. 6, p. 576-583, 1999.

BITNER, M. J.; OSTROM, A. L.; MORGAN, F.N. Service Blueprinting: A practical Technique for Service Innovation, California Management Review, 2008.

BORN, R. G.; STÅHL, I. A business course in simulation modeling, Issues in Information Systems, v. IX, n. 1, 2008.

FITZSIMMONS, J. A.; FITZSIMMONS, M. J. Administração de serviços: operações, estratégica e tecnologia da informação. 6.ed. Porto Alegre: Bookman, 2010.

GONÇALVES, J. E. L. As Empresas são Grandes Coleções de Processos. Revista de Administração de Empresas, v. 40, n. 1, p. 6-19, 2000.

GONÇALVES, J. E. L. Processo, que Processo? GV-executivo, v. 1, n. 1, 2002.

HARMON, P. Business Process Change: a guide for business managers and BPM and Six Sigma Professionals, Elsevier, 2007.

HARTVIGSEN, D. SimQuick - process simulation with Excel, 2.ed. Pearson Pretince Hall, 2005.

HILL, R. R. Process Simulation in Excel for a Quantitative Management Course, INFORMS Transactions on Education, v. 2, n. 3, 2002.

JOHANSSON, H. J. Processos de negócios. São Paulo: Pioneira, 1995.

LAGUNA, M.; MARKLUND, J. Business Process Modeling, Simulation, and Design. New Jersey: Pearson Prentice Hall, 2004

LEV, B. Book Reviews, Interfaces, v. 32, n. 5, 2002.

MAHBOUBIANA, M. Educational aspects of business simulation softwares, Procedia Social and Behavioral Sciences, v. 2, p. 5403-5407, 2010.

MEDINA, A. C.; CHWIF, L. Modelagem e Simulação de Eventos Discretos: teoria \& aplicações, $2^{\circ}$ Edição, São Paulo: Editora dos Autores, 2007.

PAIM, R., CARDOSO, V., CAULLIRAUX, H., \& CLEMENTE, R. Gestão de processos: pensar, agir e aprender. Porto Alegre: Bookman, 2009.

REIJERSA, H.A., MANSARB, S. L. Best practices in business process redesign: an overview and qualitative evaluation of successful redesign heuristics. Omega, v. 33, n.1, p. 283-306, 2005.

REIS, M. M. de O.; BLATTMANN, U. Gestão de processos em bibliotecas. Revista Digital de Biblioteconomia e Ciência da Informação, Campinas, v. 1, n. 2, p.1-17, 2004. 
STÅHL, I. Teaching simulation to business students summary of 30 years' experience, In. Proceedings of the 2007 Winter Simulation Conference, 2007.

Por solicitação da empresa analisada, os custos mostrados não são os reais, uma vez que estão multiplicados por uma constante. Entretanto, os mesmos obedecem à proporção real existente entre eles. 


\section{DADOS DOS AUTORES}

NOEL TORRES JÚNIOR` noelface@gmail.com

Doutorado em Engenharia de Produção pela USP/SP

Instituição de vinculação: Universidade Federal de Minas Gerais

Belo Horizonte/MG - Brasil

Áreas de interesse em pesquisa: Administração da Produção e Gestão de Operações em Serviços.

*Av. Antônio Carlos, 6627 Pampulha Belo Horizonte/MG 31270-901

GUILHERME GONÇALVES DE SOUZA gilhermegoncalvesdesouza@gmail.com

Graduando em Engenharia de Produçãopela UFMG

Instituição de vinculação atual: Universidade Federal de Minas Gerais

Belo Horizonte/MG - Brasil

Áreas de interesse em pesquisa: Simulação de Processos.

JÚLIA ZANDOMENICO DO NASCIMENTO juliazn@globo.com

Graduanda em Engenharia de Produção pela UFMG

Instituição de vinculação atual: Universidade Federal de Minas Gerais

Belo Horizonte/MG - Brasil

Áreas de interesse em pesquisa: Simulação de Processos. 